\title{
Orientación educativa: estrategias psicopedagógicas para el desarrollo de la autoestima en el estudiante
}

\author{
Humberto Benancio Paredes Vásquez \\ paredesvasquez1986@gmail.com \\ Universidad César Vallejo - UCV \\ Nuevo Chimbote-Perú
}

\section{RESUMEN}

La orientación educativa está dirigida a favorecer el proceso formativo de los escolares, ésta se sirve de estrategias psicopedagógicas para el desarrollo de la autoestima, que posibilita el crecimiento integral de los adolescentes. El presente trabajo tiene como propósito presentar una revisión sistematizada de la literatura científica, respecto a las diferentes estrategias psicopedagógicas, dirigidas a mejorar la autoestima de los escolares; y demostrar la importancia de la aplicación de las mismas. Para procesar la información se tomó en cuenta como metodología la exploratoria descriptiva, mediante la revisión de la literatura científica, recopilación, análisis y síntesis de datos. Dentro de las estrategias con mayor éxito descritas en la presente, se destacan: la actividad física, formación de la creencia de buenas habilidades para el éxito, el enfoque socioformativo, desarrollo de emociones en una cultura por la paz, las oportunidades de crecimiento personal, el esclarecimiento vocacional y la promoción de la salud.

Palabras Claves: orientación educativa; estrategias psicopedagógicas; autoestima. 


\title{
Educational guidance: psychopedagogical strategies for the development of self-esteem in the student
}

\begin{abstract}
The educational orientation is aimed at promoting the educational process of schoolchildren, it uses psycho-pedagogical strategies for the development of self-esteem, which enables the integral growth of adolescents. The present work aims to present a systematized review of the scientific literature, regarding the different psychopedagogical strategies, aimed at improving the self-esteem of schoolchildren; and demonstrate the importance of applying them. To process the information, the descriptive exploratory methodology was taken into account, through the review of the scientific literature, data collection, analysis and synthesis. Among the most successful strategies described herein, the following stand out: physical activity, formation of the belief of good skills for success, the socioformative approach, development of emotions in a culture for peace, opportunities for personal growth, vocational enlightenment and health promotion.
\end{abstract}

Keywords: educational orientation; psych pedagogical strategies; self-esteem

Artículo recibido: 10. Junio. 2021 Aceptado para publicación: 16. Julio. 2021 Correspondencia: paredesvasquez1986@gmail.com Conflictos de Interés: Ninguna que declarar 


\section{INTRODUCCIÓN}

Los jóvenes de la actualidad viven inmersos en ambientes sociales condicionados por la globalización, como un factor influyente cada vez más significativo, la noción de valores y cultura se entremezcla sin barreras fuera de los límites locales, lo cual a la par de traer nuevas oportunidades, trae también nuevos fenómenos sociales. La obsesión de querer tener a su alcance la cultura foránea, la moda y todas las ofertas del mundo globalizado. Todo ello, con el potencial de distorsionar la personalidad del estudiante. Así mismo, se observa que los jóvenes adolecen de buena autoestima, precisamente en la etapa que sufren transformaciones corporales y psíquicas propias de su edad, esto se evidencia en el nivel de la educación básica, donde los adolescentes, experimentan diversas experiencias, dentro de ellas despiertan las emociones, se producen cambios repentinos, y van formando su carácter, de acuerdo a sus vivencias, esta etapa es trascendental en la existencia de las personas. (Silva-Escorcia, 2015)

La familia juega un rol fundamental como el núcleo social básico, estamento donde se establece los primeros modelos de los vínculos comunitarios, donde se obtienen los valores y la personalidad, cimentados en los vínculos afectivos que permiten desde niños, niñas y adolescentes desplegarse en la colectividad. Sin embargo, existe en las instituciones educativas, adolescentes con diversas problemáticas, que se expresan en diferentes manifestaciones de conducta, cuyo factor influyente es el ámbito familiar (Ortega y Cárcamo, 2018).

Los conflictos y separaciones entre padres de familia, son las causas recurrentes de los comportamientos inadecuados de los adolescentes, resulta ser un factor dañino, que amenaza tanto su estabilidad afectiva como emocional, es muy probable, que presenten problemas de adaptación. En estos escenarios, demanda mucho tiempo para el acoplamiento en la nueva fase, se volverá un tanto difícil, entrando en un prolongado tiempo de espera. En este contexto, se evidencia como características la apatía, rehúye de los compromisos, le invade la tristeza, se encuentra preocupado, inseguro, temeroso, con sentimientos de culpa e inferioridad, maneja pocas habilidades sociales. (Panesso \& Arango, 2017)

Ante esta realidad problemática de los estudiantes, es muy importante la orientación educativa como disciplina psicopedagógica, porque contribuye al desarrollo de la buena autoestima, a través de un conjunto de estrategias de apoyo al estudiante, a lo largo de las 
diferentes etapas de su formación académica. Se hace necesario aplicar, estrategias psicopedagógicas, como el manejo de las tecnologías, para gestionar nuevos conocimientos de formación integral. Estos escenarios virtuales son una buena forma para desarrollar habilidades técnicos y sociales, que les posibilite a los estudiantes interactuar con otros para crear redes sociales, las cuales les permite nutrirse de conocimientos cada vez más veloces, mostrando un dominio admirable en la cultura digital, que les servirá como estímulo para mejorar su autoestima (Gómez, L., Valdovinos, 2020, Valvuena, 2018).

Así mismo, es muy importante introducir en la orientación educativa, como parte de las estrategias psicopedagógicas, metodologías activas que estimulen a los adolescentes, a despertar el interés por participar activamente en las diversas actividades, tanto académicas como recreativas como son los juegos lúdicos, visitas de estudio, entre otros, que ayudan al desarrollo de las interrelaciones amicales y habilidades sociales. Para que la formación sea sostenida en el tiempo, es importante, planificar, formulando objetivos claros y precisos, los cuales deben formar parte del currículo, como innovación pedagógica de cada institución educativa (Gonzáles, J., Ramírez, C., Llautong, A., Garay, G. y Sánchez, 2019).

La actual situación problemática de los escolares, nos demanda como una necesidad urgente, analizar la orientación educativa en el Currículo Nacional, así como, obtener las estrategias psicopedagógicas para desarrollar una buena autoestima, la cual proporciona información que será útil para mejorar el conocimiento y abordar el problema con propiedad. Para tal fin, se propone los objetivos de la investigación, teniendo en cuenta el propósito general, que es analizar la orientación educativa en el currículo nacional, y como objetivos específicos, analizar las causas que condicionan la baja autoestima escolar, y como respuesta a la problemática objeto de estudio, el desarrollo de estrategias psicopedagógicas para una buena autoestima.

\section{ESTRATEGIAS METODOLÓGICAS}

La búsqueda de la literatura para elaborar el artículo de revisión, se compone de tres fases: (a) búsqueda de la información, (b) organización de la información, y (c) análisis de la información. Para el proceso de la recopilación de la indagación, se determinó la metodología, consistente en la sistematización de la información científica, así como, las reglas de valoración de los artículos para la revisión documental. El texto se ha narrado, 
en forma descriptiva, porque se ajusta a la naturaleza de la investigación.(Gómez-Luna et al., 2014, Vera, 2009).

Para la organización de la información, se procedió a clasificar el material obtenido de las comunidades científicas, a través de páginas Web y otros reportes indispensables para comenzar la exploración. La estrategia para la recopilación de la información, se ha considerado, en primer orden, la búsqueda en el tesauro de la Organización de las Naciones Unidas para la educación, la Ciencia y la Cultura, posteriormente, se realizó una indagación en la base de datos de la Universidad César Vallejo, con revisiones sistemáticas de las sociedades científicas dedicadas a la psicología y a la educación. La técnica que se usó para conseguir la información fue la del fichaje, con el instrumento de autoría propia la ficha sincrética.

Para seleccionar la literatura se tuvieron en cuenta las siguientes palabras claves: autoestima, adolescencia, educación secundaria, estrategias psicopedagógicas, orientación educativa, además, se usó frases relacionadas a las palabras claves de exploración. Se analizaron las informaciones relacionadas a los escritos destacados, con el propósito de recuperar otras publicaciones que favorecen a la temática de la investigación. En la búsqueda de la información documentaria, se tomó en cuenta las informaciones provenientes de las comunidades científicas y asociaciones profesionales que recomendaban sobre la autoestima, así mismo, en los registros sistemáticos, se aplicó como regla de incorporación a los estudios realizados con adolescentes del nivel secundario, se incluyen los resultados teniendo en cuenta las recomendaciones de estrategias psicopedagógicas para la mejora de la autoestima (Andújar et al., 2012a)

Para proceder a la selección de la literatura científica, se verificaron los sumarios, en el caso de publicaciones significativas a la temática de estudio, se revisó los escritos con más amplitud, para conocer lo que contenía si estaba o no relacionado con el propósito de la investigación. Posteriormente, se redactaron en la ficha sincrética la información clasificada, parafraseando los conceptos y teorías de los autores. Para enriquecer la redacción de nuestra investigación, se hizo uso del diccionario de la Real Académica Española, instrumento que facilitó la construcción de los párrafos en un lenguaje técnico. El reporte examinado se organizó por partes: el primero referido a la baja autoestima y el segundo a las estrategias psicopedagógicas, orientadas a desarrollar una sana autoestima de los adolescentes del nivel secundario. De todo el cumulo de la información analizada, 
se sacó el reporte de diversas fuentes de las sociedades científicas, relacionadas a los descriptores objeto de estudio. En los documentos obtenidos, las temáticas sobre recomendaciones de la problemática en los adolescentes fueron: la globalización como fenómeno influyente en los adolescentes, jóvenes que adolecen de buena autoestima, la familia y su rol fundamental como núcleo social básico, los conflictos y separaciones entre padres de familia, y la orientación educativa como disciplina psicopedagógica para mejorar la buena autoestima (Andújar et al., 2012b).

Finalmente, para que la investigación tenga consistencia y garantía en la búsqueda de las revisiones, se tuvo en cuenta, autoría, año, título del artículo científico, fuente del estudio. De las publicaciones primarias se extrajo los reportes que tenían datos completos entre ellos el autor, título de la investigación científica, boletín en la que fue difundido, año de la publicación, edición, número de volumen, lugar donde se elaboró la investigación, tipo de indagación y resultado.

\section{RESULTADOS Y DISCUSIÓN}

Los escolares pasan por un periodo muy difícil en la adolescencia, donde la autoestima es disminuida por diferentes factores, una de ellos son las carencias obtenidas desde niños, que van afectando en el proceso del desarrollo de los adolescentes, es por ello, que el joven tiene dificultades para desarrollar una personalidad segura. La pérdida de su autoimagen está vinculada a la distorsión de su razonamiento, tienen una visión muy desfigurada de lo que son realmente, así lo demuestra el estudio realizado por los autores, Ceballos, G. et al., (2015), en una muestra de 242 estudiantes, el 40\% de los adolescentes percibe que tienen baja autoestima, donde el $18 \%$ corresponde al género femenino, en tanto, el $22 \%$ está relacionado al género masculino.

Existe hallazgos de altos porcentajes de escolares que presentan baja autoestima, sobre todo en lugares de la zona andina, donde pocas veces expresan con palabras lo que sienten y piensan, y si lo reconocen ante los demás, se sienten avergonzados, si lo reconoce ante sí mismo, se angustian y sufren, esto sucede con frecuencia en las zonas rurales, de acuerdo a los autores Paucar, M. y Barboza, S. (2018), revela en su investigación de una muestra de 133 estudiantes, el 80,5\% presenta baja autoestima, donde el aspecto físico es uno de los indicadores con mayor influencia negativa.

Los adolescentes que padecen sobrepeso y obesidad también se encuentran perjudicados en su autoimagen, en una muestra de 235 estudiantes el $29.3 \%$ tiene la probabilidad de 
sufrir violencia, en el género femenino de cada 10 estudiantes cinco tiende a ser agresor y a la vez víctima de la violencia. Más de la mitad de los que sufren agresiones también ejercen algún grado de agresión. El 75\% de escolares entre ser víctima de violencia y ejercer agresión se sienten afectados en su autoestima (Vilchis, 2020).

En un diagnóstico nutricional de obesidad en una muestra de 60 escolares, se analizó el resultado donde la mayoría demostraba síntomas de depresión y ansiedad. Godoy, F. (2014). Otro caso relacionado al estado nutricional, vinculado a las variables de estudio, se evaluaron un total de 146 mujeres, donde los resultados indican que las estudiantes de autoconcepto disminuido muestran altos niveles de insatisfacción física. En comparación con los resultados anteriores, existe diferencias respecto a la percepción de su estado corporal (Asuero et al., 2012).

Los factores de riesgo siempre será una constante en los estudiantes en su relación con los demás, teniendo en cuenta las recientes herramientas tecnológicas, estas han trastocado la vida de los adolescentes, en una muestra de 240 participantes, se ha podido verificar que el autoconcepto y la impulsividad cognitiva y no programada, revela que hay mayor posibilidad por la búsqueda del internet, con una tendencia a tomar decisiones rápidas, así como manifestar mayor interés por las decisiones del presente que por el futuro (Moral \& Fernández, 2019).

En otro contexto el apego a las redes sociales en relación al autoconcepto, tiene otros datos cautivadores, así se presenta en el estudio realizado con una población 152 estudiantes, llegaron a la siguiente conclusión, el escolar que tiene más acercamiento a las redes sociales, el autoconcepto disminuye más, donde el género femenino se impone con mayor porcentaje que el género masculino, en el análisis también se aprecia que no existe mayor diferencia entre grados de estudio. También se percibe que el nivel de autoestima en los diferentes grados se mantiene una tendencia levemente constante (Valdez, 2018).

Respecto a la variable de las causas que condicionan la baja autoestima, (Carrillo, 2009), afirma en su estudio realizado con una muestra de 48 adolescentes, su resultados fueron: el $27.65 \%$ tiene su autoestima baja, debido al descuido de sus progenitores, en la atención personalizada para con sus hijos, además no ofrecen seguridad, confianza, comunicación poca o casi nula, al igual los adolescentes no sienten que sus maestros, le brinden apoyo, afecto, seguridad, comunicación, en consecuencia, el adolescente tiene su autoestima 
baja, que se expresa en comportamientos de timidez, inseguridad, así como creer, que es incapaz de ser aceptado por sus compañeros, no se estiman como individuos, no les gusta su aspecto corporal, son acomplejados, no manifiestan sus emociones y sus sentimientos. El estudio de casos relacionado a la agresión filio parental de adolescentes entre los 14 y 17 años en la comunidad de Segovia, muestra que el 10\% de los hijos que incurren en actos de agresión contra sus procreadores, en otros casos oscila entre el 3\% y el 13\%, identificado como baja autoestima en los adolescentes, esto se debe al nuevo perfil educativo que presentan las familias de este tiempo, las causas que ocasionan este comportamiento agresivo con sus padres son: experiencia familiar para resolver conflictos a través de la violencia, padres excesivamente permisivos, padres sobreprotectores que satisfacen todas las necesidades de los hijos, parejas que tuvieron hijos sin planificar y expresan su enfado por el hecho ocurrido, matrimonios que sostienen una relación de enfrentamiento y suelen desacreditarse entre sus hijos. Este tipo de estilo educativo es propio de los padres de nuestra sociedad actual en la que estamos inmersos (Esquiliche Vázquez, 2016).

El suicidio que se presenta en los adolescentes se está convirtiendo en una tendencia a nivel mundial, y como característica emocional de este fenómeno es la baja autoestima, en la investigación realizada con escolares entre 13 a 18 años, y con una población 120 adolescentes, se obtuvo como resultado, que el $41 \%$ de sujetos tienen conductas suicidas, a consecuencia de los factores psicosociales que se produce en las familias disfuncionales, las que impulsan a los escolares a sufrir una depresión y una baja autoestima (Riesgos, 2018).

El fracaso académico en los escolares, es otro de los fenómenos que cobra vigencia en la actualidad, genera diversas reacciones en el educando, considerando que existen diversas causas entre ellas las situaciones psicológicas que prevalecen como factor negativo en el rendimiento escolar, el dilema se acentúa cuando el escolar repite de grado o lleva áreas atrasadas. El resultado del estudio muestra que de 120 estudiantes el 51,4\% presenta baja autoestima, como producto del bajo rendimiento académico (Ferrel Ortega et al., 2014). Entre las diversas estrategias psicopedagógicas para mejorar la autoestima, la actividad física es una buena terapia no solo para mejorar la salud, sino también para mejorar la presentación personal, ya que controla el sobrepeso. El estudio aplicado a 90 estudiantes de los grados de tercero a quinto de la educación básica, el $50 \%$ presentaba bajos niveles 
de autoestima que se le atribuye a la escasa actividad física, por el contrario, los estudiantes que frecuentemente realizan sus actividades físicas mejoraron su autoconcepto (Lizarazo López et al., 2020).

Desarrollar en el escolar la creencia y expectativa alta de que tiene buenas habilidades para el éxito, que es un pilar de importancia proponerse con entusiasmo, es potenciar la autoeficacia como estrategia de seguridad y confianza en el adolescente, el acompañamiento debe ser permanente estimulando y resaltando las acciones sobresalientes. El estudio de escolares de primero a cuarto de secundaria se registró que la autoeficacia fortalece la autoestima significativamente. Esto en relación al género, los niveles de autoestima mejor establecido fueron en los varones. (Diego García Álvarez, María José Soler, 2019).

Las propuestas metodológicas activas, basadas en técnicas creativas para enseñar y aprender conceptos, tiene impacto en los disidentes en el proceso de enseñanza aprendizaje, que fortalece la autoestima de los escolares. La aplicación de las estrategias se realizó con estudiantes de 15 a 16 años, incentivando la curiosidad en la creatividad al estudiar en las diferentes áreas, los resultados muestran cambios notables en las estrategias de aprendizaje. Además, se percibe favorablemente en el resultado académico y en el crecimiento de la autoestima escolar (Sánchez, I. y Pulgar, J. 2017).

La orientación educativa como disciplina psicopedagógica, aporta significativamente a la mejora de la autoestima escolar, para ello es primordial aplicar un conjunto de estrategias psicopedagógicas, durante todo el proceso de su formación académica. Según los resultados aplicados por un equipo de psicopedagogos, encontraron que en su mayoría realizaban un tratamiento clínico, que no ha dado buenos resultados, por lo que se recomienda encaminar por un trabajo de carácter psicopedagógico en la línea del enfoque socioformativo. (De la Oliva, D., Tobón, S., Pérez, A., Romero, J., Escamilla, K. 2020) Promover la orientación educativa desarrollando competencias emocionales por la paz, es un verdadero reto en la educación secundaria, las instituciones educativas que se proponen transmitir en su práctica como una acción formadora en los estudiantes y familias se ha convertido en una verdadera revolución educativa. El estudio de un grupo de 36 profesionales de la orientación educativa, tuvieron como resultado, los cambios en la forma de planear las clases en el aula, promoviendo una educación inclusiva, justa, 
equitativa y no violenta, se logró modificar la conducta de los estudiantes, convirtiendo el centro educativo en un espacio de paz. (Sánchez, B. y Escobedo, 2019).

La fase de formación académica debería ser un espacio educativo, donde se brinde una serie de opciones, estrategias y oportunidades de crecimiento personal y social, necesarias para desarrollar habilidades que permitan hacer frente a las dificultades que se presentan en la comunidad en futuros escenarios. La referencia investigativa refiere que la orientación educativa está planteada para unir visiones y enfoques positivos en función de atender las diversas necesidades de los escolares, con el fin de generar bienestar en los educandos (Del Carmen Rodríguez Esquivel \& Gallardo Córdova, 2020).

Para que el estudiante tenga la claridad ante una decisión, es necesario de la orientación profesional, en tal sentido, el estudio realizado sobre el esclarecimiento vocacional, manifiesta que, el escolar necesita de la inducción de los psicopedagogos, para encausar la orientación hacia la carrera profesional de acuerdo a sus capacidades, a partir del acondicionamiento psicológico, pedagógico, científico y metodológico de acuerdo a la vocación según resultados en la intervención a cada estudiante (Rojas Valladares, Adalia; Torres Zerquera, Leticia del Carmen; Pérez Egües, 2020).

Una de las condiciones del ser humano para que sea feliz es tener vitalidad, para ello, el centro escolar es un laboratorio para desarrollar buenos hábitos, función que, la orientación educativa direcciona en el proceso sobre los cuidados básicos que un estudiante debe manejar en la promoción de la salud. En una investigación, cuya finalidad fue resaltar el rol de los profesionales en la orientación educativa y vocacional en la promoción de la salud, la información fue categórica, al manifestar que, es muy necesario contar con espacios grupales para que se desarrollen los estamentos de salud pública y privada, en el marco de un bienestar sano del ciudadano (Montserrat \& Carbajal Arregui, 2020).

\section{DISCUSIÓN}

Los resultados encontrados en este estudio en cuanto a la aplicación de estrategias psicopedagógicas para mejorar la autoestima, muestran en general que los adolescentes de familias disfuncionales tienen un autoconcepto disminuido por diversas causas: el aspecto físico es uno de los indicadores con mayor influencia negativa sobre todo en la zona andina del Perú, se sienten avergonzados y con sentimientos de inferioridad respecto a los adolescentes de la zona costa, otros factores como la obesidad, ser víctimas de la 
violencia, el descuido de sus progenitores, la agresión filio parental, el fracaso académico, y las conductas suicidas, influyen fuertemente en una conducta de baja autoestima. Estos resultados coinciden con la literatura de Bermúdez, V. (2018), quien manifiesta que la "adolescencia es una etapa de turbulencias y cambios a nivel físico, socioafectivo y conductual, caracterizada por una elevada reactividad emocional, relacionada directamente a los diversos problemas de los adolescentes" que todo ello puede originar problemas de carácter psicológicos.

En los resultados del trabajo se pudo apreciar que la actividad física, es una terapia no sólo para tener una buena salud, sino también para mejorar la presentación personal ante los demás, así como, desarrollar en los escolares la creencia y expectativa alta de que tiene buenas habilidades para el éxito, esto significa, potenciar la autoeficacia como estrategia de seguridad y confianza. Otro aspecto que se ha convertido en una revolución educativa, es el desarrollo de competencias emocionales por la paz, que proponen transmitir en sus prácticas como una acción formadora tanto en los estudiantes como en las familias.

También se tiene resultados como, las estrategias y oportunidades de crecimiento personal y social, que son otras habilidades para tenerlo en cuenta en la formación integral del escolar, porque desarrolla mecanismos personales, que le permite hacer frente a las dificultades que se presentan en la comunidad en futuros escenarios. En este marco, la inducción de los psicopedagogos, también juegan un rol muy importante para encausar la orientación hacia la carrera profesional de acuerdo a sus capacidades, es muy positivo y motivador el esclarecimiento vocacional en los estudiantes.

Con la finalidad de dar respuestas a los problemas de baja autoestima, se ha tenido en cuenta a un grupo de psicopedagogos que realizaron un estudio de salud mental, en casos de tratamiento clínico con jóvenes de secundaria, los resultados no fueron lo que se esperaba, ante esto, se recomienda encaminar por un trabajo de carácter psicopedagógico en la línea del enfoque socioformativo, que ha dado buenos resultados, por tanto, se recomienda aplicar las estrategias activas socioemocionales, para ayudar a desarrollar una autoestima positiva en los adolescentes.

En otros estudios dan a conocer que, entre las estrategias psicopedagógicas, la orientación educativa direccionada en el proceso sobre los cuidados básicos que un estudiante debe manejar en la promoción de la salud, es muy significativo, así lo reporta la información 
en forma categórica, al manifestar que, es muy necesario contar con espacios grupales para que se desarrollen los estamentos de salud pública y privada, en el marco de un bienestar de los individuos, sabiendo que, las condiciones del ser humano para que sea feliz es tener vitalidad, en este contexto, los estudiantes podrán mejorar significativamente su autoestima.

\section{CONCLUSIÓN O CONSIDERACIONES FINALES}

1) La orientación educativa en el currículo nacional, está dirigido a orientar el trabajo pedagógico para desarrollar las habilidades de los escolares, de acuerdo a las exigencias del contexto. La planificación está orientada de acuerdo a las características psicológicas de los escolares según los ciclos de cada nivel. Así mismo, tiene las directivas para el encauzamiento de los enfoques transversales, la organización y planificación de los lineamientos pedagógicos en un constructo de orientación formativa integral.

2) Los estudios refieren que las causas que ocasiona la baja autoestima escolar, se encuentran caracterizados según su contexto, así tenemos: disfunción familiar, bullying, abuso sexual, la obesidad, agresión en los infantes, conflictos de pareja, desorden nutricional, problemas de comunicación, problemas de violencia, la crianza represiva de los padres, padres permisivos, discriminación social, la apariencia física.

3) Las estrategias psicopedagógicas, aporta significativamente a la mejora de la autoestima escolar, para ello es primordial aplicar un conjunto de estrategias socioemocionales, durante todo el proceso de su formación académica. Para que tenga éxito la intervención del plan operativo en la orientación educativa, es fundamental, seleccionar las estrategias que más les gusta a los adolescentes entre ellos, los escenarios virtuales, los cuales en esta época se han convertido en un medio indispensable para su relación con los demás. En este contexto, los tutores de cada sección constituyen un eslabón vital en el sistema de formación integral.

\section{LISTA DE REFERENCIAS}

Andújar et al., (2012a). Evidencia científica y recomendaciones sobre cribado de agudeza visual. Revisión bibliográfica. Universidad de alicante. https://www.mscbs.gob.es/biblioPublic/publicaciones/recursos_propios/resp/revi sta_cdr 
Andújar et al., (2012b). Evidencia científica y recomendaciones sobre cribado de agudeza visual. Revisión bibliográfica. Universidad de alicante. https://www.mscbs.gob.es/biblioPublic/publicaciones/recursos_propios/resp/revi sta_cdr

Barrett PM, Webster HM y Wallis JR (1999). Adolescent self-esteem and cognitive skills training: A school-based intervention. Journal of Child and Family Studies, 8, 217-227.

Bermúdez, V. (2018), Ansiedad, depresión, estrés y autoestima en la adolescencia. relación, implicaciones y consecuencias en la educación privada. https://institucional.us.es/revistas/cuestiones/26/03.\%20MO\%2003_26\%20DEFI NITIVO.pdf

Carrillo, L. (2009), La familia, la autoestima y el fracaso escolar del adolescente. Tesis Doctoral. Editorial de la Universidad de Granada. https://digibug.ugr.es/bitstream/handle/10481/2150/17811089.pdf?sequence=1\& is Allowed=y

Ceballos, G., Suárez, Y., Suescún, J., Gamarra, L., Gonzales, K. y Sotelo, A. (2015), Ideación suicida, depresión y autoestima en adolescentes escolares de Santa Marta, Revista Duazary, Vol. $12 \quad-\quad \mathrm{N}^{\circ}$ 1, 15-22. file://C:/Users/LENOVO/Downloads/1394-Texto\%20del\%20art\%C3\%ADculo3069-1-10-20150410\%20(1).pdf

Chamorro, M. (2016). Percepciones frente al divorcio de los padres y nivel de adaptabilidad en un grupo de adolescentes de Trujillo. Pueblo Cont. Vol. 27 (1). Pág. 192.: http://journal.upao.edu.pe/PuebloContinente/article/viewFile/405/370

De la Oliva, D., Tobón, S., Pérez, A., Romero, J., Escamilla, K. (2020). Evaluación del modelo educativo constructivista de orientación educativa e intervención psicopedagógica desde el enfoque socioformativo. Vol. 55 - $\mathrm{N}^{\circ}$ 2, pp.561-576. https://www.raco.cat/index.php/Educar/article/view/359310?

Doyle AB y Markiewicz D (2005). Parenting, marital conflict and adjustment from earlyto midadolescence: Mediated by adolescent attachment style? Journal of Youth and Adolescence, 34, 97-110.

Dubois DL y Tevendale HD (1999). Self-esteem in childhood and adolescence: Vaccine or epiphenomenon? Applied and Preventive Psychology, 8, 103-117. 
Eskin M, Ertekin K y Demir H (2008). Efficacy of a problem-solving therapy for depression and suicide potential in adolescents and young adults. Cognitive Therapy and Research, 32, 227-245.

Esquiliche, M. (2015). Maltrato de menores (14 - 17 años) a sus padres en el ámbito familiar. Número 4. Universidad Nacional de Educación a Distancia (UNED). https://revistas.proeditio.com/ehquidad/article/view/940/976

Ferrel, F., Vélez, J. y Ferrel, L. (2014). Factores psicológicos en adolescentes escolarizados con bajo rendimiento académico: depresión y autoestima. Revista Encuentros, Universidad Autónoma del Caribe, 12 (2), pp. 35-47. http://eds.b.ebscohost.com/eds/pdfviewer/pdfviewer?vid=0\&sid=c3db6fd154f6-4123-893b-d30e41d210ec\%40pdc-v-sessmgr06

Garaigordobil M (2002). Assessment of an intervention of social behaviour, intra-group relations, selfconcept and prejudiced cognitions during adolescence. International Journal of Psychology and Psychological Therapy, 2, 1-22.

Garber J, Robinson NS y Valentiner D (1997). The relation between parenting and adolescent depression: Self-worth as a mediator. Journal of Adolescent Research, $12,12-33$.

García, D., Soler, M. y Cobo, R. (2019). Bienestar psicológico en adolescentes: relaciones con autoestima, autoeficacia, malestar psicológico y síntomas depresivos. Revista de Orientación Educacional, 33(63), 23-43, 2019.

http://eds.a.ebscohost.com/eds/pdfviewer/pdfviewer?vid=0\&sid=f6a447e1-7f624861-970c-53558542c4f1\%40sdc-v-sessmgr03

Godoy, F. (2014). Sintomatologia de depresión, ansiedad y baja autoestima en mujeres obesas con trastorno del comedor compulsivo. Rev. chil. nutr. vol.41 no.3 Santiago Sept. 2014.

https://scielo.conicyt.cl/scielo.php?script=sci_arttext\&pid=S0717$75182014000300005 \& \operatorname{lng}=\mathrm{en} \& \ln \mathrm{l}=\mathrm{en} \#$ ?

Gómez, Luna y otros (2014). Metodología para la revisión bibliográfica y la gestión de información de temas científicos, a través de su estructuración y sistematización. Dyna, vol. 81, núm. 184, abril, 2014, pp. 158-163 Universidad Nacional de Colombia Medellín, Colombia. Recuperado de: 
https://www.redalyc.org/articulo.oa?id=49630405022om/vol86/vol186_6/RS866 C_575.pdf

Gonzales, J., Ramírez, C., Llautong, A., Garay, G. y Sánchez, A. (2019). La estrategia psicopedagógica lúdica. Revista atlante: Cuaderno de Educación y Desarrollo. En línea: https://www.eumed.net/atlante/2019/06/estrategia-psicopedagógicaludica.html.

Huang JS, Norman GJ, Zabinski MF, Calfas KJ y Patrick K (2007). Body image and selfesteem among adolescents undergoing an intervention targeting dietary and physical activity behaviors. Journal of Adolescent Health, 40, 245-51.

Isasa, M. y Carbajal, M. (2020). Abordaje preventivo de la orientación educativa y vocacional en Uruguay. Volumen $20 \mathrm{~N}^{\circ} 1$ - Facultad de Psicología Universidad Nacional de La $\quad$ Plata. http://eds.a.ebscohost.com/eds/pdfviewer/pdfviewer?vid=0\&sid=9ecc64da-fe2f4a7a-a1cf-721bb4329efa\%40sessionmgr4006

Lizarazo, L., Burbano, V. y Valdivieso, M. (2020). Correlación entre actividad física y autoestima de escolares adolescentes: un análisis de tipo trasversal. Revista Virtual Universidad Católica del Norte, (60), 95-115. http://eds.a.ebscohost.com/eds/pdfviewer/pdfviewer?vid=0\&sid=03916ab934b7-4f51-b358-ac3eba8dc4e6\%40sessionmgr4007

Mestre V y Frías D (1996). La mejora de la autoestima en el aula. Aplicación de un programa para niños en edad escolar (11-14 años). Revista de Psicología General y Aplicada, 49, 279-290.

Moral, M. (2018). Uso problemático de internet en adolescentes españoles y su relación con autoestima e impulsividad. Avances en Psicología Latinoamericana, vol. 37, núm.

2019.http://eds.b.ebscohost.com/eds/pdfviewer/pdfviewer?vid=0\&sid=da43fa3f82f0-407c-a447-ea587dae3bbf\%40pdc-v-sessmgr06

Ortega, M. y Cárcamo, H. (2027). Relación familia escuela en el contexto rural. Miradas desde las familias. http://www.scielo.org.pe/pdf/educ/v27n52/a06v27n52.pdf Panesso, K. \& Arango, M. (2017). La autoestima, proceso humano. Revista electrónica. PSYCONEX. Departamento de Psicología. Medellín, Colombia. Vol. 9 - № 14. 
Recuperado de: dile://C:/Users/LENOVO/Downloads/328507Texto\%20del\%20art_culo-129909-1-10-20170803.pdf

Paucar, M. y Barboza, S. (2017), Niveles de autoestima de los estudiantes de la Institución educativa 9 de diciembre del Pueblo Nuevo de Chincha. Tesis para optar el Título de segunda especialidad profesional de psicología educativa.

Petty KH, Davis CL, Tkacz J, Young-Hyman D yWaller J (2009). Exercise effects on depressive symptoms and self-worth in overweight children: A randomized controlled trial. Journal of Pediatric Psychology, 34, 929-939.

Revista Prisma $N^{\circ} 23$ Adolescencia y Riesgos, (2018). Inclusión Educativa: Factores Psicosociales Asociados a Conductas Suicidas en Adolescentes - pp. 185-207. http://eds.b.ebscohost.com/eds/pdfviewer/pdfviewer?vid=0\&sid=0b1c056c4302-4e19-820a-e44cd72fc7eb\%40pdc-v-sessmgr02

Robins RW y Trzesniewski KH (2005). Self-esteem development across the lifespan. Current Directions in Psychological Science, 14, 158-162.

Rodríguez, N. y Gallardo, K. (2020). El bienestar y la orientación educativa enfocados en las nuevas generaciones. Vol. $31, \quad \mathrm{n}^{\circ} 2,2^{\circ}, \quad \mathrm{pp} . \quad 7-18$. http://eds.a.ebscohost.com/eds/pdfviewer/pdfviewer?vid=0\&sid=1a93398ee138-42f2-9d5f-88023a02aff4\%40sessionmgr4007

Rodríguez, C. y Caño, A. (2012), Autoestima en la adolescencia: análisis y estrategia de intervención. International Journal of Psychology and Psychological Therapy, 12, 3, 389-403. file:///C:/Users/LENOVO/Downloads/DialnetAutoestimaEnLaAdolescencia-4019787.pdf

Rojas, A., Torres, L. y Pérez, M. (2020). El proceso de orientación hacia la carrera, desde el rol del psicopedagogo en la institución educativa. Revista Conrado, 16(73), 3540. https://conrado.ucf.edu.cu/index.php/conrado/article/view/1267/1262

Sánchez, I. y Pulgar, J. (2017). Impacto de una renovación metodológica en física bajo técnicas creativas en las estrategias de aprendizaje y la autoestima. Revista Paradigma, Vol. XXXVIII, N. ${ }^{\circ}$ 2, diciembre de 2017 / 184 - 204. http://eds.a.ebscohost.com/eds/pdfviewer/pdfviewer?vid=0\&sid=27306392c289-4a5b-b3e8-a670c517295a\%40sessionmgr4008

Sánchez, B. y Escobedo, S. (2019). Educación emocional para la paz. Una propuesta para la práctica en la orientación educativa. Facultad de Educación, Universidad 
Complutense de Madrid. Vol. 19, $\mathrm{N}^{\circ}$ 81, pp. 67-68. http://eds.a.ebscohost.com/eds/pdfviewer/pdfviewer?vid=0\&sid=d3034c127dd6-4494-9d35-03a7d90eec51\%40sessionmgr4008

Silva, I. \& Mejía, I. (2015), Autoestima, adolescencia y pedagogía. Revista electrónica educare. EISSN: 1409-4258 Vol. 19(1) ENERO-ABRIL, 2015: 241256.https://studylib.es/doc/7745918/autoestima--adolescencia-ypedagog\% $\mathrm{C} 3 \% \mathrm{ADa}$

Tashakkori A, Thompson VD, Wade J y Valente E (1990). Structure and stability of selfesteem in late teens. Personality and Individual Differences, 11, 885-893.

Trzesniewski KH, Donnellan MB y Robins RW (2003). Stability of self-esteem across the life span. Journal of Personality and Social Psychology, 84, 205-220.

Valbuena, L. (2018). Estrategias psicopedagógicas para comprender la autoagresión y la construcción del joven en la era digital. http://repositorio.ucundinamarca.edu.co/handle/20.500.12558/1619

Valdez, A. (2018). Adicción a Redes Sociales y Autoestima en adolescentes de Nivel Secundario de una Institución Privada. http://tesis.ucsm.edu.pe/repositorio/handle/UCSM/7785\#?

Vera, O. (2009), Artículos de revisión. Cómo escribir artículos de revisión. Rev. Med. La $\begin{array}{lllll}\text { Paz: } & 15(1): & 63 & - & 69 .\end{array}$ http://www.scielo.org.bo/pdf/rmcmlp/v15n1/v15n1_a10.pdf

Vilchis, E. (2020). Agresión, victimización y autoestima en escolares con sobrepeso y obesidad. RevistaCONAMED.Vol.25Núm.2,2020•pp57-65. http://eds.b.ebscohost.com/eds/pdfviewer/pdfviewer?vid=0\&sid=9dc3a895858d-4ac5-a65c-cdae25f8e34b\%40pdc-v-sessmgr03 